\title{
Evaluation of Holonomic Quantum Computation: Adiabatic Versus Nonadiabatic
}

\author{
LiXiang Cen, ${ }^{1}$ XinQi Li, ${ }^{1}$ YiJing Yan, ${ }^{2}$ HouZhi Zheng, ${ }^{1}$ and ShunJin Wang ${ }^{3}$ \\ ${ }^{1}$ NLSM, Institute of Semiconductors, The Chinese Academy of Sciences, Beijing 100083, People's Republic of China \\ ${ }^{2}$ Department of Chemistry, Hong Kong University of Science and Technology, Kowloon, Hong Kong \\ ${ }^{3}$ Department of Physics, Sichuan University, Chengdu 610031, People's Republic of China
}

(Received 14 September 2002; published 10 April 2003)

\begin{abstract}
Based on the analytical solution to the time-dependent Schrödinger equations, we evaluate the holonomic quantum computation beyond the adiabatic limit. Besides providing rigorous confirmation of the geometrical prediction of holonomies, the present dynamical resolution offers also a practical means to study the nonadiabaticity induced effects for the universal qubit operations.
\end{abstract}

DOI: $10.1103 /$ PhysRevLett.90.147902

PACS numbers: 03.67.Lx, 03.65.Vf

Recently, a remarkable contribution termed as a holonomic approach to quantum computation [1] has been proposed and received increasing interest. Transcending the traditional dynamical means the holonomic quantum computation encodes and processes information in a degenerated eigenspace of the governing Hamiltonian. The intrinsic nontrivial global holonomies, either Abelian or non-Abelian [2,3], are exploited by driving the system to undergo appropriate loops in the parameter space adiabatically. Besides suggesting an intriguing connection between the gauge fields and the information processing, such a geometrical means for quantum manipulation is believed to have built-in fault-tolerant features $[4,5]$ due to its inherent stability against local perturbations. Considerable attention has been addressed to this topic recently and the all-geometrical implementation for universal quantum gates was proposed with optical schemes, based on laser manipulation of ions confined in a Paul trap [6] or neutral atoms in an optical resonator [7].

The very existence of the proposed holonomic quantum computation relies strictly on adiabatic evolution processes, in which the time-evolution ray and the curvature of the ray space are naturally defined, and the associating holonomy can thus be considered as a dynamically irrelevant (i.e., pure geometrical) property. However, the dynamics processes in reality could not be ideally adiabatic. The inevitable nonadiabaticity will alter the track of the time-evolution ray and thus induce deviation from the adiabatic consequences. The evaluation of such deviation and the resulting errors in quantum computation is clearly a dynamical problem that goes beyond the geometrical exploration.

In this Letter, we shall employ the typical processes of the optical scheme to exploit this subject. For each gate operation, we will choose an appropriate loop of the Hamiltonian in the parameter space, and then solve analytically the associating time-dependent Schrödinger equation by virtue of the cranking representation or gauge transformation approach. It will show that the resulting analytical dynamics solution not only naturally recovers the geometrical consequence of holonomic transformation, including the simple Abelian phase factor and the general non-Abelian operation, but also naturally provides a practical mean for evaluating the nonadiabaticity induced errors in qubit operations.

Consider the standard optical scheme explored recently for the holonomic quantum computation [6,7], in which a qubit is encoded in a four-level $\Lambda$-type trapped ion (or a similar cavity atom). The three ground levels $\left|g_{i}\right\rangle(i=$ $1,2,3)$ are highly degenerated and each couples to the excited state $|e\rangle$ in a tunable manner. The states $\left|g_{1}\right\rangle$ and $\left|g_{2}\right\rangle$ stand for the computational basis states $|0\rangle$ and $|1\rangle$, respectively, and $\left|g_{3}\right\rangle$ is an ancillary level required for gate operations. Such a system admits two dark states that have no contributions from the excited state. The holonomic quantum computation is realized here as the adiabatic passage via the dark states as follows [6,7]. Through changing the Rabi frequencies to drive the dark states to undergo appropriate cyclic evolutions in an adiabatic fashion, the universal single-qubit gate operations $\left(e^{i \phi|1\rangle(1 \mid}\right.$ and $\left.e^{i \phi \sigma_{y}}\right)$ and the controlled two-qubit phase shift gate operation $\left(e^{i \phi|11\rangle|11|}\right)$ can be generated from the global geometry associated with the bundle of the eigenspace of dark states [6]. Note that such a geometrically established framework is inherently fragile. The timeevolution, i.e., the time-dependent Schrödinger equation, involves all four levels of the system. In other words, the dynamical nonadiabaticity induces a certain coupling of the dark states with other states, leading to two types of error in quantum computing. One is the leakage of the dynamical state out of the computational space and another is the remaining deviation from the desired transformation. In the following, we shall explore both of them in detail for each individual gate operation.

Let us start with the single-qubit gate operation $e^{i \phi|1\rangle 11}$. Its dynamical evolution in the proposed optical scheme is specified by the periodic Hamiltonian $[6,7]$

$$
H(t)=\Omega \sin \theta\left(\sigma_{2 e}+\sigma_{e 2}\right)+\Omega \cos \theta\left(\sigma_{3 e} e^{i \varphi}+\sigma_{e 3} e^{-i \varphi}\right),
$$

with $\sigma_{j e}=\left|g_{j}\right\rangle\langle e|(j=2,3)$. Here, $\theta$ and $\varphi$ are the controllable loop parameters denoting, respectively, the relative 
amplitude and the phase of Rabi frequency. Consider the loop evolution in which $\varphi=\gamma t$ rotates at a constant frequency $\gamma$, while $\theta$ remains for the time being as a fixed parameter. It is well known that, in the geometrical exploration of adiabatic limit [2], the dark state, $|D(t)\rangle=$ $\cos \theta\left|g_{2}\right\rangle-\sin \theta e^{i \gamma t}\left|g_{3}\right\rangle$, of the system acquires a net Berry phase as $|D(T)\rangle=e^{i \phi}|D(0)\rangle$ with $\phi=4 \pi \sin ^{2} \theta$, after a period $T=2 \pi / \gamma$. To the dynamical resolution beyond the adiabatic limit, one should solve the Schrödinger equation $i \partial_{t}|\Psi(t)\rangle=H(t)|\Psi(t)\rangle$. The analytical solution to the dynamics is as follows. We use the cranking representation, in which the Hamiltonian of Eq. (1) can be cranked as

$$
H(t) \equiv e^{i \gamma t \sigma_{33}} H_{0} e^{-i \gamma t \sigma_{33}} .
$$

The unitary transformation $e^{i \gamma t \sigma_{33}}$ can be viewed as an element of the $\mathrm{SU}(3)$ group, and

$$
H_{0}=\Omega \sin \theta\left(\sigma_{2 e}+\sigma_{e 2}\right)+\Omega \cos \theta\left(\sigma_{3 e}+\sigma_{e 3}\right),
$$

which is time independent. Consequently, the dynamical invariant of the system can be shown as

$$
I(t)=e^{i \gamma t \sigma_{33}}\left(H_{0}+\gamma \sigma_{33}\right) e^{-i \gamma t \sigma_{33}}=H(t)+\gamma \sigma_{33},
$$

which satisfies [8] $d I(t) / d t=\partial I(t) / \partial t-i[I(t), H(t)]=0$. The term $\gamma \sigma_{33}$ in Eq. (4) accounts for an extra gauge potential since $H(t)$ depends on time explicitly. Now the recurrent basis $|\psi(t)\rangle$ of the system, differing from the basic solution $|\Psi(t)\rangle$ of the Schrödinger equation only by a phase factor, can be obtained by solving the instantaneous eigensolutions of $I(t)$. The characteristic equation turns out to be

$$
x^{3}-(\gamma / \Omega) x^{2}-x+(\gamma / \Omega) \sin ^{2} \theta=0 .
$$

The eigenvalues of $I(t)$ relate to its roots as $E_{i}=\Omega x_{i} ; i=$ $-1,0,1$. Note that the state $\left|g_{1}\right\rangle$ is decoupled from the others [cf. Equation (1)]. It is straightforward to show that the recurrent basis $\left|\psi_{0}(t)\right\rangle$ associated with the eigenvalue $E_{0}$ approaches to the dark state $|D(t)\rangle$ in the adiabatic limit of $\gamma / \Omega \rightarrow 0$. However, the nonadiabatic $\left|\psi_{0}(t)\right\rangle$ contains in general also the excited state $|e\rangle$ component. Reported in Fig. 1(a) is the fidelity, defined here as

$$
\eta(\theta, \gamma / \Omega)=\left|\left\langle\psi_{0}(0) \mid D(0)\right\rangle\right|^{2}=\left|\left\langle\psi_{0}(T) \mid D(T)\right\rangle\right|^{2} .
$$

Thus, $(1-\eta)$ measures the nonadiabaticity induced leakage error. Besides the leakage, the nonadiabatic evolution will result in a modified phase factor in comparing with its adiabatic counterpart. Instead of the net Berry phase, the cyclic evolution here induces a total phase

$$
\Phi(\theta, \gamma / \Omega)=\int_{0}^{T}\left\langle\psi_{0}(t)\left|i \partial_{t}-H(t)\right| \psi_{0}(t)\right\rangle d t=2 \pi \frac{E_{0}}{\gamma} .
$$

This is the so-called Lewis-Riesenfeld phase by which $\Psi(t)=e^{i \Phi} \psi(t)$. Note that in the adiabatic $(\gamma / \Omega \rightarrow 0)$ limit, one can obtain via the derivative of Eq. (5) that $E_{0} / \gamma \equiv x_{0} \Omega / \gamma \rightarrow 2 \sin ^{2} \theta$, and Eq. (7) recovers the Berry phase, $\Phi_{\text {ad }}=4 \pi \sin ^{2} \theta$. The nonadiabatically modified phase $\Phi(\theta, \gamma / \Omega)$ is shown in Fig. 1(b).
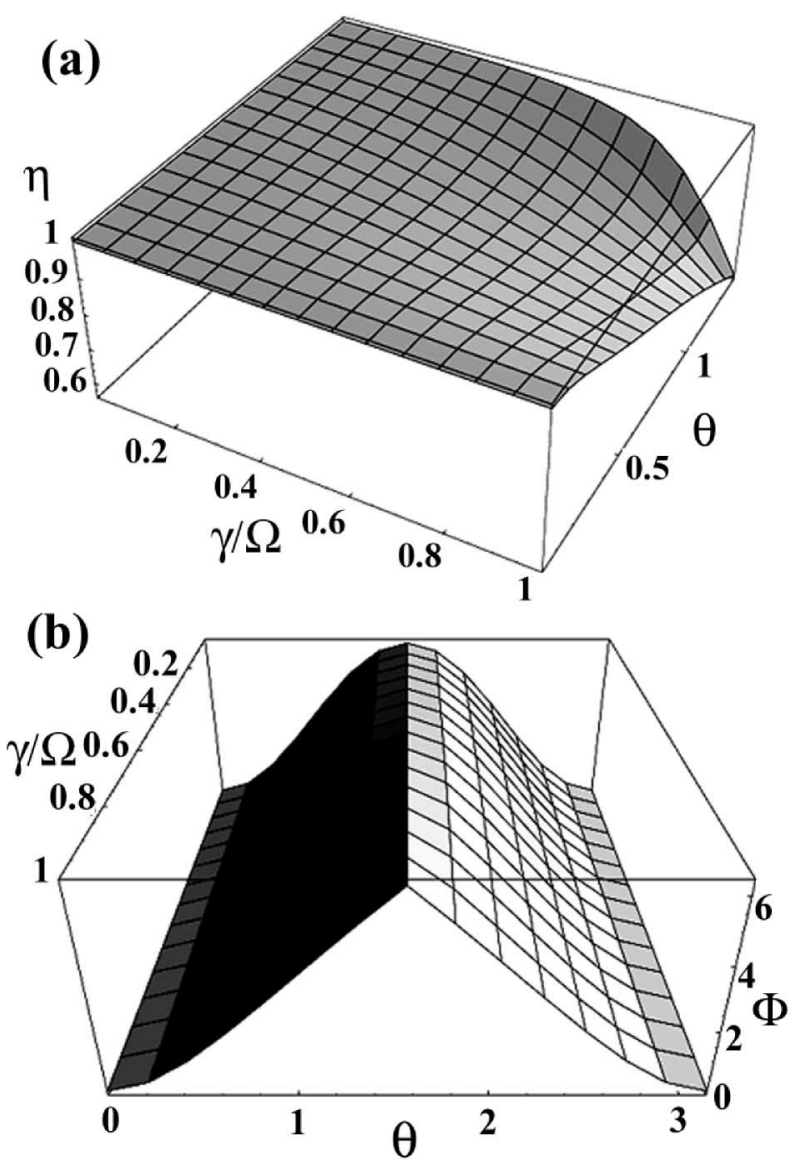

FIG. 1. The nonadiabatic effects on the single-qubit gate $e^{i \phi|1\rangle\langle 1|}$, reported in terms of (a) the fidelity parameter $\eta$ [Eq. (6)] and (b) the modified phase $\Phi$ [Eq. (7)], as functions of $(\theta, \gamma / \Omega)$. Note that this figure can also serve for the nonadiabatic effects on the controlled two-qubit phase shift gate [cf. comments following Eq. (19)].

The validity of the above evaluation arguments is based on a presumption that the initial state $|D(0)\rangle$ can be generated from the computational basis $\left|g_{2}\right\rangle$ and vice versa. Explicitly, such processes can be accomplished by the driven Hamiltonian Eq. (1) through changing the parameter $\theta$ adiabatically. Usually, the nonadiabatically varied $\theta$ shall also induce error for the quantum computation. In the following, we shall show that this error can in principle be eliminated through a matching interaction to compensate the induced gauge potential term. Specifically, one can use the following Hamiltonian (setting $\varphi=0$ ):

$$
H_{\mathrm{tot}}(t)=H(t)+H^{\prime}(t), \quad H^{\prime}(t)=i \dot{\theta}(t)\left(\sigma_{23}-\sigma_{32}\right) .
$$

It follows that the dynamical invariant of the system $H_{\text {tot }}(t)$ has now the form of $I(t)=H(t)$; thus, the above state transformation can be processed without inducing additional error. The compensating interaction $H^{\prime}(t)$ can in principle be realized by using a microwave field, with 
its intensity accurately being controlled through a derivative feedback process, to couple the two nearly degenerate levels $\left|g_{2}\right\rangle$ and $\left|g_{3}\right\rangle$.

Now turn to the evaluation of gate operation $e^{i \phi \sigma_{y}}$. Let us explore the evolution generated by the Hamiltonian

$$
\begin{aligned}
H(t)= & \Omega \sin \theta\left[\cos \varphi\left(\sigma_{1 e}+\sigma_{e 1}\right)+\sin \varphi\left(\sigma_{2 e}+\sigma_{e 2}\right)\right] \\
& +\Omega \cos \theta\left(\sigma_{3 e}+\sigma_{e 3}\right),
\end{aligned}
$$

with $\varphi \equiv \gamma t$. It is known that the adiabatic cyclic evolution of the Hamiltonian generates a non-Abelian holonomy due to its degeneracy structure of the dark states. It can be easily worked out from the formula of Ref. [3] that the holonomic transformation is

$$
u_{C}=e^{i 2 \pi \cos \theta D_{y}},
$$

where $D_{y}=i\left(\left|D_{2}\right\rangle\left\langle D_{1}|-| D_{1}\right\rangle\left\langle D_{2}\right|\right)$. The two dark states, $\left|D_{1}\right\rangle=\cos \theta\left|g_{1}\right\rangle-\sin \theta\left|g_{3}\right\rangle$ and $\left|D_{2}\right\rangle=\left|g_{2}\right\rangle$, span the degenerate space of the starting (ending) Hamiltonian. $H(t)$ of Eq. (9) possesses an su(4) Lie algebraic structure whose dynamical resolution is usually very complicated. Surprisingly, as we shall show in the following, this time-dependent system can be solved exactly, leading thus to the analytical evaluation of its adiabatic and nonadiabatic properties.

Instead of the cranking method used earlier, we introduce here the unitary gauge transformation [9], $\left|\Psi^{g}(t)\right\rangle \equiv$ $G^{-1}(t)|\Psi(t)\rangle$, with $G(t)=e^{-\gamma t\left(\sigma_{12}-\sigma_{21}\right)}$. The covariant Schrödinger equation can be obtained as $i \partial_{t}\left|\Psi^{g}(t)\right\rangle=$ $H^{g}\left|\Psi^{g}(t)\right\rangle$, in which the gauged Hamiltonian is given by

$$
\begin{aligned}
H^{g}= & G^{-1} H G-i G^{-1} \partial G / \partial t \\
= & \Omega \sin \theta\left(\sigma_{1 e}+\sigma_{e 1}\right)+\Omega \cos \theta\left(\sigma_{3 e}+\sigma_{e 3}\right) \\
& +i \gamma\left(\sigma_{12}-\sigma_{21}\right) .
\end{aligned}
$$

As it is time independent, the basic solutions $\left\{\left|\Psi_{n}^{g}(t)\right\rangle\right\}$ to the above covariant equation can be easily obtained. The corresponding eigenvalues are summarized as follows:

$$
\begin{aligned}
& E_{1,2}= \pm 2^{-1 / 2} \bar{\Omega}\left[1-\sqrt{1-4(\gamma / \Omega)^{2} \cos ^{2} \bar{\theta}}\right]^{1 / 2}, \\
& E_{3,4}= \pm 2^{-1 / 2} \bar{\Omega}\left[1+\sqrt{1-4(\gamma / \Omega)^{2} \cos ^{2} \bar{\theta}}\right]^{1 / 2},
\end{aligned}
$$

in which $\bar{\Omega}=\sqrt{\Omega^{2}+\gamma^{2}}, \quad$ and $\quad \cos \bar{\theta}=(\cos \theta) /$ $\left[1+(\gamma / \Omega)^{2}\right]$. With the analytical solutions of $\left\{\left|\Psi_{n}^{g}(t)\right\rangle\right\}$, the dynamical basis of Eq. (9) can be directly obtained as $\left|\Psi_{n}(t)\right\rangle=G(t)\left|\Psi_{n}^{g}(t)\right\rangle=e^{-i E_{n} t} G(t)\left|\Psi_{n}(0)\right\rangle$, from which one can see that $E_{n}$ has the natural implication related to the total phase. The evolution operator generated by the Hamiltonian [Eq. (9)] can then be obtained accordingly via $U(t)=\sum_{n}\left|\Psi_{n}(t)\right\rangle\left\langle\Psi_{n}(0)\right|$.

Before presenting the nonadiabaticity induced effects, let us outline the asymptotic behavior of the evolution in the adiabatic limit. It follows that $\lim _{\gamma / \Omega \rightarrow 0} E_{1,2} / \gamma=$ $\pm \cos \theta$. The associating phase-equipped dynamical basis states, resulted as the limits of corresponding equations, assume the form (noting that $\varphi \equiv \gamma t$ )

$$
\begin{aligned}
\left|\Psi_{1,2}^{\mathrm{ad}}(t)\right\rangle= & 2^{-1 / 2} e^{\mp i \varphi \cos \theta}\left[(\cos \theta \cos \varphi \pm i \sin \varphi)\left|g_{1}\right\rangle\right. \\
& \left.+(\cos \theta \sin \varphi \mp i \cos \varphi)\left|g_{2}\right\rangle-\sin \theta\left|g_{3}\right\rangle\right] .
\end{aligned}
$$

Note here that $\left|\Psi_{1,2}^{\text {ad }}(0)\right\rangle=\left(\left|D_{1}\right\rangle \mp i\left|D_{2}\right\rangle\right) / \sqrt{2}$. One can verify that $\left|\Psi_{1}^{\text {ad }}(t)\right\rangle$ and $\left|\Psi_{2}^{\text {ad }}(t)\right\rangle$ in Eq. (13) are the instantaneous eigenstates associated with the degenerated eigenvalue 0 of the Hamiltonian Eq. (9), and the equipped phases are just the Berry phases accordingly. Thus, the cyclic evolution restricted to the space spanned by these two degenerated states, assuming the form of

$$
\begin{aligned}
U_{\mathrm{ad}}(T)= & e^{-i 2 \pi \cos \theta}\left|\Psi_{1}^{\mathrm{ad}}(0)\right\rangle\left\langle\Psi_{1}^{\mathrm{ad}}(0)\right| \\
& +e^{i 2 \pi \cos \theta}\left|\Psi_{2}^{\mathrm{ad}}(0)\right\rangle\left\langle\Psi_{2}^{\mathrm{ad}}(0)\right|,
\end{aligned}
$$

is purely geometrical. It is easily recognized that $U_{\text {ad }}(T)$, obtained here as the limiting case of the dynamical solution, is identical to the $u_{C}$ [Eq. (10)] obtained from holonomic transformation. Thus, the above discussion has also provided an example of dynamical verification of the celebrated geometric prediction of non-Abelian holonomy [3].

We reemphasize here that the analytical solutions to the general, nonadiabatic basis set $\left\{\left|\Psi_{n}(t)\right\rangle ; n=1, \ldots, 4\right\}$ can be obtained for this system [Eq. (9)], and Eq. (13) are just the adiabatic limits of two corresponding equations. The fidelity $\eta$, by which $1-\eta$ measures the nonadiabaticity induced leakage out of the computational space, can now be defined by the projection [cf. Equation (6)]

$$
\eta(\theta, \gamma / \Omega)=\sum_{i=1}^{2}\left|\left\langle D_{i}|U(T)| \Psi(0)\right\rangle\right|^{2} .
$$

Figure 2 depicts in detail the dark state population transfer (the solid curves) from the initial state $|\Psi(0)\rangle=\left|g_{2}\right\rangle$ as a function of $\{\theta, \gamma / \Omega\}$, and the fidelity of the nonadiabatic evolution (the dash curves).

Further, to have the required transform between the computational basis $\left|g_{1}\right\rangle$ and the dark state $\left|D_{1}\right\rangle$, one needs to let the parameter $\theta$ in Eq. (9) tunable at $\varphi=0$. Similar to the former case, we have here [cf. Equation (8)]

$H_{\mathrm{tot}}(t)=H(t)+H^{\prime}(t), \quad H^{\prime}(t)=i \dot{\theta}(t)\left(\sigma_{13}-\sigma_{31}\right)$.

Again, the added $H^{\prime}$ is to compensate the varying $\theta$ induced gauge potential.

We are now in the position to investigate the dynamical consequence of a controlled two-qubit phase shift gate, $e^{i \phi|11\rangle\langle 11|}$. In contact to the geometrical implementation proposed in Ref. [6], the proposed scheme there exploited two-color biexciton laser manipulation on systems such as trapped ions [10]. Briefly, the two-qubit manipulation is realized by applying two bichromatic laser pairs that drive resonantly and cooperatively with the two-photon/ biexciton transitions, $\left|g_{2} g_{2}\right\rangle \leftrightarrow|e e\rangle$ and $\left|g_{3} g_{3}\right\rangle \leftrightarrow|e e\rangle$, respectively. However, each laser individually is operated in the off-resonant regime with respect to a single 


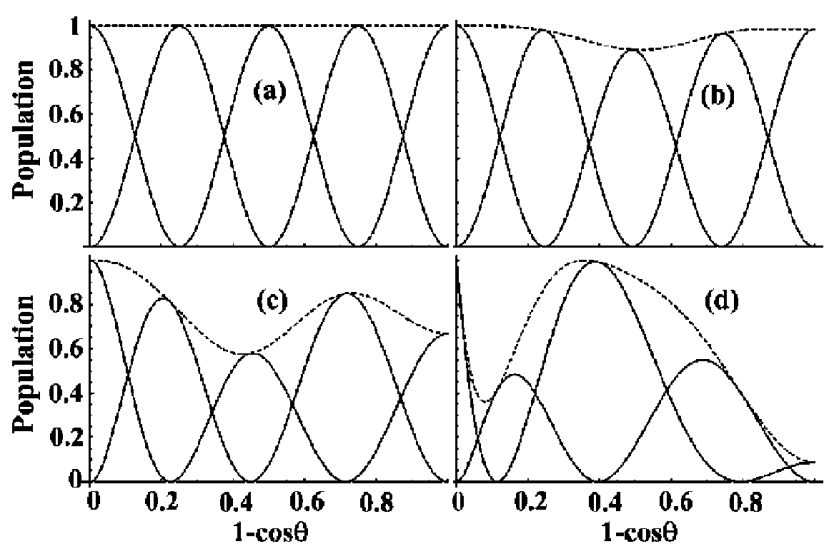

FIG. 2. The nonadiabatic effects on the single-qubit gate $e^{i \phi \sigma_{y}}$ in terms of the dark state population transfer (solid curves) for various values of nonadiabaticity: $\gamma / \Omega=0.01$, $0.2,0.5$, and 0.8 for (a), (b), (c), and (d), respectively. The initial state assumes $\left|D_{2}\right\rangle$. Included are also the fidelity $\eta$ [Eq. (15)] parameter (dash curves).

excitation. The system can be described, under a welldefined condition, by the Hamiltonian [6]

$$
\begin{aligned}
H_{\text {eff }}= & -\Omega_{1}^{\text {eff }}\left(e^{i 2 \varphi_{1}}|e e\rangle\left\langle g_{2} g_{2}\right|+\text { H.c. }\right) \\
& +\Omega_{2}^{\text {eff }}\left(e^{i 2 \varphi_{2}}|e e\rangle\left\langle g_{3} g_{3}\right|+\text { H.c. }\right),
\end{aligned}
$$

where the relative intensity of the effective Rabi frequencies $\tan \theta=-\Omega_{1}^{\text {eff }} / \Omega_{2}^{\text {eff }}$ and the phase difference $\varphi / 2=$ $\varphi_{1}-\varphi_{2}$ are tunable. One can see that the basis states $\left|g_{1} g_{1}\right\rangle,\left|g_{1} g_{2}\right\rangle$, and $\left|g_{2} g_{1}\right\rangle$ are decoupled from the evolution, while $\left|g_{2} g_{2}\right\rangle$ that serves as the code $|11\rangle$ evolves in the closed subspace spanned by $\left\{\left|g_{2} g_{2}\right\rangle,\left|g_{3} g_{3}\right\rangle,|e e\rangle\right\}$. Introducing the $\mathrm{su}(3)$ generators explicitly,

$$
\begin{aligned}
& A_{e 2}=e^{i 2 \varphi_{1}}|e e\rangle\left\langle g_{2} g_{2}\left|, \quad A_{e 3}=e^{i 2 \varphi_{1}}\right| e e\right\rangle\left\langle g_{3} g_{3}\right|, \\
& A_{23}=\left|g_{2} g_{2}\right\rangle\left\langle g_{3} g_{3}\right|, \quad A_{\mu \nu}^{\dagger}=A_{\nu \mu},
\end{aligned}
$$

Eq. (17) can then be recast as

$$
\begin{aligned}
H_{\text {eff }}= & {\left[\left(\Omega_{1}^{\text {eff }}\right)^{2}+\left(\Omega_{2}^{\text {eff }}\right)^{2}\right]^{1 / 2}\left[\sin \theta\left(A_{2 e}+A_{e 2}\right)\right.} \\
& \left.+\cos \theta\left(A_{3 e} e^{i \varphi}+A_{e 3} e^{-i \varphi}\right)\right] .
\end{aligned}
$$

Obviously, this Hamiltonian possesses an su(3) algebraic structure isomorphic to that of Eq. (1). Thus, all the discussions therein and Fig. 1 as well, provided $\Omega=$ $\left[\left(\Omega_{1}^{\text {eff }}\right)^{2}+\left(\Omega_{2}^{\text {eff }}\right)^{2}\right]^{1 / 2}$, also hold for the present system.

In conclusion, we have evaluated the holonomic quantum computation beyond adiabatic limitation. The nonadiabaticity induced effects in terms of the leakage error and the modified phase are evaluated by exploring the typical optical process and solving exactly the timedependent Schrödinger equation for each qubit operation. In a realistic scheme of geometric/holonomic quantum computation, the evaluation of imperfections is necessary, particularly since a direct extension of the adiabatic scenario to the nonadiabatic situation is not attainable [11]. Note that the nonadiabatic effects on the Abelian holonomy were exploited before in the context of general quantum dynamics [12]. The present work constitutes the first exploration of the nonadiabatic effects in the context of quantum computation, covering over both the Abelian holonomy and its much more complicated non-Abelian counterpart. For the two-qubit operation, we adopt a simplified model [Eq. (17)] in which other competing second-order optical processes involved in the single trapped ion are neglected. The resulting two-qubit operation is shown isomorphic to the Abelian holonomic realization of single-qubit gate $e^{i \phi|1\rangle\langle 1|}$, and its dynamical consequences are therefore also studied accordingly. The inclusion of other competing processes, such as twophoton (Rayleigh) scattering that may interfere with the biexcitonic dynamics, and their effects on the holonomic realization of the two-qubit gate will be studied in the future.

This work is supported by the Postdoctoral Science Foundation, the special funds for Major State Basic Research Project No. G001CB3095, the NNSF No. 10175029 of China, and the RGC of Hong Kong Government.

[1] P. Zanardi and M. Rasetti, Phys. Lett. A 264, 94 (1999); J. Pachos, P. Zanardi, and M. Rasetti, Phys. Rev. A 61, 010305(R) (2000); J. Pachos and S. Chountasis, Phys. Rev. A 62, 052318 (2000); J. Pachos and P. Zanardi, Int. J. Mod. Phys. B 15, 1257 (2001).

[2] M. V. Berry, Proc. R. Soc. London A 392, 45 (1984); B. Simon, Phys. Rev. Lett. 51, 2167 (1983).

[3] F. Wilczek and A. Zee, Phys. Rev. Lett. 52, 2111 (1984).

[4] J. Preskill, in Introduction to Quantum Computation and Information, edited by Hoi-Kwong Lo, S. Popescu, and T. Spiller (World Scientific, Singapore, 1999).

[5] J. A. Jones, V. Vedral, A. Ekert, and G. Castagnoli, Nature (London) 403, 869 (2000); A. Ekert et al., J. Mod. Opt. 47, 2501 (2000); G. Falci, R. Fazio, G. M. Palma, J. Siewert, and V. Vedral, Nature (London) 407, 355 (2000).

[6] L. M. Duan, J. I. Cirac, and P. Zoller, Science 292, 1695 (2001).

[7] A. Recati, T. Calarco, P. Zanardi, J. I. Cirac, and P. Zoller, Phys. Rev. A 66, 032309 (2002).

[8] H. R. Lewis, Jr., Phys. Rev. Lett. 18, 510 (1967).

[9] S. J. Wang, F. L. Li, and A. Weiguny, Phys. Lett. A 180, 189 (1993); S. J. Wang et al. (unpublished).

[10] K. Molmer and A. Sorensen, Phys. Rev. Lett. 82, 1835 (1999).

[11] L. X. Cen, X. Q. Li, and Y. J. Yan, quant-ph/0208119.

[12] Y. Aharonov and J. Anandan, Phys. Rev. Lett. 58, 1593 (1987); S. J. Wang, Phys. Rev. A 42, 5107 (1990); S. L. Zhu and Z. D. Wang, Phys. Rev. B 61, 1142 (2000). 